\title{
Hábitos alimenticios de Caranx vinctus (Perciformes: Carangidae), especie de importancia comercial en la bahía de Acapulco, Guerrero, México
}

María Isabel Vázquez-Ozuna1, Genoveva Cerdenares-Ladrón de Guevara², Agustín Aucencio Rojas-Herrera ${ }^{1 *}$, Juan Violante-González ${ }^{1}$, Sergio García-Ibañez $^{1}$

\& Víctor Manuel Rosas-Guerrero ${ }^{3}$

1. Facultad de Ecología Marina, Universidad Autónoma de Guerrero, Gran Vía Tropical, número 20, Fraccionamiento Las Playas, 39390, Acapulco, Guerrero, México; mivo2065@gmail.com, rojash56@hotmail.com, viojuang@yahoo. com.mx, sergariba@yahoo.com.mx

2. Universidad del Mar, Campus Puerto Ángel Cd Universitaria, 70902, Municipio San Pedro Pochutla, Oaxaca, México; gcerdenares@angel.umar.mx

3. Escuela Superior en Desarrollo Sustentable, Universidad Autónoma de Guerrero, Técpan de Galeana, Guerrero, México; victor_rosas@yahoo.com

* Correspondencia

Recibido 03-II-2020. Corregido 14-IV-2020. Aceptado 13-V-2020.

\begin{abstract}
Abtract: Feeding habits of Caranx vinctus (Perciformes: Carangidae), a commercially important species in Acapulco Bay, Guerrero, Mexico. Introduction: Caranx vinctus is an important species in local fisheries, used as a source of protein for human consumption, however, its trophic ecology is little known on the Mexican Pacific coast. Objective: Know the feeding habits of $C$. vinctus. Methods: The stomach contents of 268 specimens, captured beach nets, in the Playa Las Hamacas cooperative society, in Acapulco Bay, Guerrero, Mexico, their food items were analyzed. The prey-specific index of relative importance (\% PSIRI) was applied to determine the main food categories (breadth was calculated using Levin's index. Possible differences in the diet by sex, sexual maturity, or season were identified through Morisita Horn index and a multivariate ANOSIM analysis. Results: Crustaceans mainly represented by megalopa larvae of Portunoidae (16.51\%), Teleostei (12.7 $\%)$, unidentified organic matter (11.2\%), and Anchoa ischana (10.6\%) were the most important food items. The Levin index indicates low amplitude of the trophic niche $(\mathrm{Bi}=0.29)$ so $C$. vinctus can be considered as a specialist predator with marked preferences towards some food items. The Morisita Horn index indicated that there was an overlap between sexes (females and males $C \lambda=0.84$ ), but not between sexual maturity (Juveniles and adults $C \lambda=0.56$ ) and seasons (Rainy and dry $C \lambda=0.03$ ). ANOSIM analysis indicated that there were significant differences in the diet of $C$. vinctus by sex, sexual maturity, and season. Conclusion: It was established that in general C. vinctus is a zoplantophage and ichthophagus species (Trophic Level $=3.39$ ).
\end{abstract}

Key words: jack; Carangidae; diet; trophic level; Portunoidae; Anchoa ischana; Tropical Eastern Pacific.

Vázquez-Ozuna, M.I., Cerdenares-Ladrón de Guevara, G., Rojas-Herrera, A.A., ViolanteGonzález, J. García-Ibañez, S., \& Rosas-Guerrero, V.M. (2020). Hábitos alimenticios de Caranx vinctus (Perciformes: Carangidae), especie de importancia comercial en la bahía de Acapulco, Guerrero, México. Revista de Biología Tropical, 68(3), 752-764.

La doradita Caranx vinctus Jordan \& Gilbert, 1882 pertenece a la familia Carangidae y se distribuye en el Pacífico oriental tropical, desde la costa occidental de Baja California, México hasta Perú en aguas costeras y oceánicas (Allen \& Robertson, 1978; Robertson \& Allen, 2015). Esta especie presenta una importancia económica en pesquerías locales y de sustento, alcanza una talla máxima de $37 \mathrm{~cm}$ de longitud total (LT) y, se captura 
principalmente con redes de enmalle, línea de mano y con arpón (Smith-Vaniz, 1995; EspinoBarr, Cruz, \& García, 2003; Gutiérrez-Zavala \& Cabrera-Mancilla, 2019).

Las especies pertenecientes al género Caranx son consideradas como depredadores piscívoros, sin embargo, también pueden alimentarse de crustáceos y en menor medida de moluscos y otros invertebrados (Silvano, 2001; Sley, Jarboui, Ghorbel, \& Bouain, 2008; Saucedo-Lozano et al. 2012). En algunas especies se ha determinado que la dieta durante la etapa juvenil se basa en el consumo de crustáceos y durante el crecimiento aumenta el consumo de peces hasta la fase adulta (Sley, Jarboui, Ghorbel, \& Bouain, 2009).

Además, cabe destacar la importancia que tienen los carangidos como parte de la dieta de grandes depredadores como tiburones, peces pico, dorados y túnidos (Oxenford \& Hunte, 1999; Hoffmayer \& Parsons, 2003; Júnior, Vooren, \& Lessa, 2004; Estupiñan-Montaño, Cedeño-Figueroa, \& Galván-Magaña, 2009). Por lo que, su rol como mesodepredadores hace que una gestión sustentable de estos recursos sea esencial para la integridad del ecosistema, ya que al ocupar niveles tróficos intermedios en la cadena trófica permiten la canalización de energía desde los niveles tróficos bajos a los altos y viceversa (Cury et al. 2000), lo que implicaría que, presiones pesqueras fuertes o condiciones ambientales desfavorables hacia estos, podría tener un impacto sobre los demás componentes del ecosistema. Pese a su relativa importancia, no existen estudios detallados, a nivel cuantitativo, sobre su alimentación; al respecto, de la Cruz-Agüero, Arellano Martínez, Cota Gómez, \& de la Cruz-Agüero (1997) mencionan que, para Baja California Sur, México, C. vinctus se alimenta principalmente de pequeños peces, crustáceos e invertebrados bentónicos.

En términos generales, los estudios de alimentación representan un elemento importante en el conocimiento de la biología básica de las especies, permitiendo conocer el papel que estas desempeñan a nivel ecológico, el uso del hábitat, la disponibilidad y variación de las especies presa, así como las relaciones intra e interespecíficas entre los diferentes componentes del ecosistema (principalmente competencia y depredación) (Yáñez-Arancibia \& Nugent, 1977; Hajisamaea, Choua, \& Ibrahim, 2003; Correa, Albrecht, \& Hahn, 2011; Ramos, D.M.A., Ferreira, A., dos Santos, N., Cunha, R.E.M.C., \& Machado, 2015). Esta información también puede ayudar a la gestión del ecosistema, ya que se puede utilizar para construir modelos tróficos (Dantas, Barletta, Ramos, Lima, \& da Costa, 2013) o ser aplicado a estudios de ecología trófica que incluyen cambios espaciales, estacionales y ontogenéticos en la dieta de las especies (Guedes, Araujo, Pessanha, \& Milagre, 2015). Dado lo anterior, el presente trabajo tiene como objetivo principal determinar los hábitos alimentarios de $C$. vinctus, considerando posibles diferencias en cuanto a sexo, madurez sexual y época, así como también su nivel trófico y amplitud del nicho.

\section{MATERIALES Y MÉTODOS}

Sitio de estudio: Todos los especímenes analizados fueron tomados de las capturas comerciales artesanales que realizan los pescadores en la costa de fondo blando en la Bahía de Acapulco, Guerreo, en el Pacífico oriental tropical de México. Los especímenes fueron capturados mediante el arte de pesca, chinchorro playero, en la sociedad cooperativa Playa las Hamacas. Se recolectaron peces de manera quincenal capturados entre marzo 2017 y abril 2018.

Recolección de datos: A cada espécimen se le tomaron las siguientes medidas: longitudtotal (LT) y peso total (W), con precisiones respectivas de $1 \mathrm{~mm}$ y $0.01 \mathrm{~g}$.

Los contenidos estomacales fueron recolectados y congelados para posteriormente determinar los diferentes componentes de la dieta de la especie.

Cada componente se identificó hasta el mínimo taxón posible, con la ayuda de claves específicas (Keen, 1958; Mauchline, 1971; Brusca, 1980; Modlin, 1984; Hendrickx \& 
Salgado-Barragán, 1991; Fischer et al., 1995; Allen \& Robertson, 1998; García-Madrigal, 2007). Cada componente se pesó con una balanza electrónica de $0.01 \mathrm{~g}$ de precisión.

Análisis de datos: Para determinar el número de estómagos necesarios para caracterizar la dieta general y la dieta de las diferentes categorías (sexos, madurez sexual y épocas), se realizaron curvas acumuladas de especies con EstimateS (Colwell, 2016). Estas curvas se construyeron a partir del valor del índice de diversidad de Shannon-Wiener (H') y el número de estómagos. Dentro del cálculo de las curvas, un coeficiente de variación $(\mathrm{CV})$ igual o inferior a $5 \%$ (0.05) indicó que el número de estómagos fue el adecuado para representar la dieta de acuerdo a trabajos publicados (Jiménez-Valverde \& Hortal, 2003; Moreno-Sánchez et al., 2019).

Para cada ítem alimenticio se calculó su índice numérico, índice gravimétrico e índice de aparición (Hynes, 1950; Hyslop, 1980). Para valorar de manera integral la importancia que tiene cada presa en la dieta de $C$. vinctus se utilizó el Índice de Importancia Relativa Específica de la Presa (\% PSIRI), propuesto por Brown, Bizzarro, Cailliet, \& Ebert (2012) el cual se expresa de la siguiente manera:

$$
\% \mathrm{PSIRI}_{\mathrm{i}}=\frac{\% \mathrm{FO}_{\mathrm{i}} \times\left(\% \mathrm{PN}_{\mathrm{i}}+\% \mathrm{PW}_{\mathrm{i}}\right)}{2}
$$

donde \% FOi es el porcentaje de la frecuencia de ocurrencia, y expresa las veces en que una presa apareció en la dieta de un depredador con respecto al número total de estómagos que contenían alimento; $\% \mathrm{PNi}=\sum_{j=1}^{n} \% \mathrm{Nij} / \mathrm{ni}$ es el porcentaje de la Abundancia Específica de la Presa en Número de organismos y $\% \mathrm{PWi}=\sum_{j=1}^{n} \% \mathrm{Wij} / \mathrm{ni}$ el porcentaje de la Abundancia Específica de la Presa en peso, en donde: \% Nij es la abundancia en número de la categoría de presas $\mathrm{i}$ en la muestra de un estomago $\mathrm{j}, \%$ Wij es el porcentaje de la abundancia en peso de la categoría de presas i en la muestra de un estomago j y ni es el número de estómagos que contiene una presa i. Este índice es aditivo con respecto al nivel taxonómico y permite comparaciones entre estudios.

La determinación de la amplitud de la dieta se utilizó para evaluar de manera cuantitativa si los organismos presentan un comportamiento generalista (presentan una alimentación variada sin ninguna selección) o especialista (presentan una preferencia por cierta presa). Se utilizó el índice de Levin (Krebs, 1999), cuando Bi se acerca a 0 se considera especialista y cuando se acerca a 1 es generalista.

Para evaluar el traslape de dietas entre sexos (hembra y machos), grado de madurez sexual (juveniles y adultos) y temporada (lluvias y secas) (tutiempo.net, 2019), se utilizó la rutina para superposición del nicho de Morisita-Horn (Horn, 1966; Smith, \& Zaret, 1982). El valor de este índice varía entre cero cuando las dietas son completamente distintas y uno, cuando las dietas son idénticas o similares. Se considera un traslape biológico significativo cuando el valor excede a 0.60 ; y valores menores a 0.60 se considera que no hay traslape (Langton, 1982).

El análisis de una vía de similitud (ANOSIM) con 999 permutaciones, se utilizó para probar la hipótesis nula de que no hay diferencias en la composición de la dieta entre sexos (hembras y machos), madurez sexual (juvenil y adulta) y diferentes estaciones (seca y lluviosa). Los análisis se realizaron utilizando el paquete estadístico PRIMER 6 (Clarke \& Warwick, 2001). Para determinar la posición trófica de C. vinctus se utilizó el método propuesto por Cortés (1999), esto es:

$$
\mathrm{NT}_{\mathrm{i}}=1+\left(\sum_{\mathrm{j}=1}^{\mathrm{n}} \mathrm{P}_{\mathrm{j}} \times \mathrm{NT}_{\mathrm{j}}\right)
$$

Donde $\mathrm{Pj}$ es la proporción que cada categoría de presa tiene en la dieta del depredador; y NTj es el nivel trófico de cada categoría de presa $\mathrm{j}$. Los niveles tróficos de presas se obtuvieron de la base de datos del Proyecto Sea Around Us (Pauly, Zeller, \& Palomares, 2020). 


\section{RESULTADOS}

Se recolectaron 278 estómagos de los cuales $268(96.4 \%)$ presentaron alimento y 10 (3.59 \%) se encontraron vacíos. Con respecto al sexo, el $60.24 \%$ fueron hembras y $39.75 \%$ machos. El intervalo de tallas de los organismos analizados fluctuó entre 16 y $27.5 \mathrm{~cm}$ LT y el peso total entre 49 y $250 \mathrm{~g}$. Según el CV de la curva de acumulación de presas, el tamaño de muestra fue suficiente para describir la dieta de Caranx vinctus de la muestra general y el tamaño de la muestra por categorías (sexo, madurez sexual y temporada) fueron adecuados para describir la dieta de esta especie (Tabla 1).

El análisis de la dieta permitió identificar 24 ítems presa, clasificados en tres grandes grupos taxonómicos: peces (cuatro ítems), crustáceos (21 items) y moluscos (dos ítems) (Tabla 2).

De acuerdo a la frecuencia de aparición, Teleostei represento el $26.97 \%$, larvas megalopa de Portunoidae el $22.85 \%$, materia orgánica no identificada (MONI) $13.48 \%$ y Anchoa ischana (Jordan \& Gilbert, 1882) el $11.24 \%$.

TABLA 1

Tamaño mínimo de muestra de Caranx vinctus de la Bahía de Acapulco, determinado para todas las muestras y por sexo, madurez sexual y época

TABLE 1

Minimum sample size of Caranx vinctus from Acapulco Bay, Mexico, determined for all samples and by sex, sexual maturity, and season

\begin{tabular}{lccc}
\multicolumn{1}{c}{ Categoría } & Ns & Nsm & CV \\
General & 268 & 98 & $\leq 0.05$ \\
Hembras & 147 & 76 & $\leq 0.05$ \\
Machos & 97 & 90 & $\leq 0.05$ \\
Juveniles & 173 & 92 & $\leq 0.05$ \\
Adultos & 94 & 83 & $\leq 0.05$ \\
Lluvias & 143 & 56 & $\leq 0.05$ \\
Secas & 125 & 115 & $\leq 0.05$ \\
\hline
\end{tabular}

Ns = número de estómagos analizados, Nsm = número mínimo de estómagos por categoría, $\mathrm{CV}=$ coeficiente de variación por tamaño de muestra.

$\mathrm{Ns}=$ number of stomachs analyzed, $\mathrm{Nsm}=$ minimum number of stomachs by category, $\mathrm{CV}=$ coefficient of variation for the respective sample size.
Numéricamente se cuantificaron un total de 4461 organismos, correspondiendo a las larvas megalopa de Portunoidae el $33.45 \%$, larvas mysis de Penaeoidea un $12.4 \%$, Euphausia sp. represento el $12.26 \%$ y el mísido Archaeomysis sp. represento el $9.39 \%$.

Las presas en los estómagos acumularon un peso de $164.9 \mathrm{~g}$ de acuerdo al método gravimétrico, los componentes más importantes fueron en los Crustacea, larvas mysis de Penaeoidea con $30.27 \%$ y Archaeomysis sp. con el $16.29 \%$, Anchoa ischana el $7.85 \%$ y peces Teleostei representaron el $6.31 \%$.

En lo que respecta al \% PSIRI, se encontró que megalopas de Portunoidae representaron el $16.51 \%$, seguido de Teleostei con $12.7 \%$, materia orgánica no identificada (MONI) con el $11.2 \%$, Anchoa ischana con el $10.6 \%$. Estos cuatro componentes conformaron el $51.01 \%$.

Para las hembras se registraron 147 estómagos con alimento, en los cuales, los ítems más importantes fueron: Archaeomysis sp. (20.7 \%), larvas megalopa de Portunoidae (20.62\%), larvas mysis de Penaeoidea (17.55 $\%)$, Anchoa ischana (6.21\%) y otros Teleostei (5.75\%). Para los machos se registraron 97 estómagos con alimento, se encontró que los ítems más importantes del espectro trófico fueron; larvas mysis de Penaeoidea (30.01\%), otros Crustacea (14.81\%), larvas megalopa de Portunoidae (11.87\%) y Mollusca Gatropoda (7.98 \%) (Fig. 1). Se presentaron 24 ejemplares de $C$. vinctus con los estómagos con alimento, a los cuales no fue posible sexar por su incipiente desarrollo gonádico. Se determinó que la proporción de hembras y machos fue significativamente diferente de $1: 1(\mathrm{X} 2=\mathrm{P}>0.05)$, la proporción fue de 1:0.65.

En el análisis de la alimentación por madurez sexual, los ejemplares juveniles de C. vinctus $(\mathrm{n}=173)$ tuvieron un total de 19 componentes alimentarios. Dónde los más importantes de acuerdo con el \% PSIRI fueron: larvas megalopa de Portunoidae (25.02 \%) seguido por larvas mysis de Penaeoidea (19.11\%), Archaeomysis sp. (15.34\%) y otros Crustacea (12.85\%), constituyendo el 72.35 $\%$ del \% PSIRI. Para los ejemplares adultos de 
TABLA 2

Espectro trófico de Caranx vinctus en la Bahía de Acapulco

TABLE 2

Trophic spectrum of Caranx vinctus in Acapulco Bay

\begin{tabular}{|c|c|c|c|c|c|c|c|}
\hline Especies presa & $\% \mathrm{FA}$ & $\% \mathrm{PN}$ & $\% \mathrm{~N}$ & $\% \mathrm{PW}$ & $\% \mathrm{~W}$ & $\%$ PSIRII & NT \\
\hline \multicolumn{8}{|l|}{ Gastropoda } \\
\hline Turritellidae & 4.49 & 33.00 & 1.37 & 30.5 & 3.29 & 1.4 & 2.1 \\
\hline Littorinidae & 3.00 & 8.1 & 1.03 & 8.0 & 2.97 & 0.2 & 2.1 \\
\hline \multicolumn{8}{|l|}{ Crustacea } \\
\hline Infraclase Cirripedia & 0.75 & 45.8 & 0.09 & 26.5 & 0.00 & 0.3 & 2.52 \\
\hline Huevo de Copepoda & 2.25 & 97.2 & 0.31 & 91.7 & 0.01 & 2 & 1 \\
\hline Subclase Copepoda & 0.37 & 100 & 0.02 & 100 & 0.00 & 0.37 & 2.2 \\
\hline Squilla sp. & 1.50 & 16.2 & 0.13 & 17.7 & 0.24 & 0.19 & 3.5 \\
\hline \multicolumn{8}{|l|}{ Mysidae } \\
\hline Acanthomysis sp. & 1.12 & 100 & 0.67 & 100 & 0.28 & 1.12 & 2.2 \\
\hline Archaeomysis sp. & 6.74 & 84.9 & 9.39 & 84.6 & 16.29 & 5.5 & 2.2 \\
\hline \multicolumn{8}{|l|}{ Amphipoda } \\
\hline Amphilochidae & 0.37 & 50 & 0.04 & 50 & 0.00 & 0.2 & 1 \\
\hline Hyperiidae & 1.12 & 35.1 & 1.37 & 38.6 & 0.07 & 0.4 & 2.2 \\
\hline Isopoda & 0.75 & 41.7 & 0.07 & 41.7 & 0.06 & 0.3 & 2.2 \\
\hline Euphausia sp. & 10.49 & 96.8 & 12.26 & 97.2 & 3.07 & 9.8 & 2.2 \\
\hline \multicolumn{8}{|l|}{ Decapoda } \\
\hline Larvas mysis & 8.61 & 72.3 & 12.44 & 70.7 & 30.27 & 5.4 & 2.2 \\
\hline Postlarvas & 5.62 & 77.3 & 8.34 & 54 & 2.10 & 2.8 & 2.2 \\
\hline Axiidae sp. & 0.37 & 10 & 0.02 & 2.7 & 0.02 & 0.1 & 2.52 \\
\hline Zoea de Porcellanidae & 9.36 & 75.2 & 5.60 & 67 & 2.89 & 7.7 & 2.2 \\
\hline Megalopa de Porcellanidae & 3.75 & 16.7 & 0.40 & 37.6 & 1.33 & 0.9 & 2.2 \\
\hline Zoea de Portunoidea & 7.12 & 70.3 & 7.53 & 63 & 2.66 & 3.1 & 2.2 \\
\hline Megalopa de Portunidae & 22.85 & 87.1 & 33.45 & 73.9 & 9.73 & 16.51 & 2.2 \\
\hline \multicolumn{8}{|l|}{ Actinopterygii } \\
\hline Teleostei & 26.97 & 43.5 & 2.31 & 57.9 & 6.31 & 12.7 & 3.24 \\
\hline Larvas de Teleostei & 2.25 & 37.3 & 0.45 & 42.4 & 0.72 & 0.9 & 2.2 \\
\hline Larva de Scianidae & 1.87 & 61.4 & 0.25 & 76.6 & 3.90 & 1.2 & 2.2 \\
\hline Anchoa ischana & 11.24 & 98.3 & 1.64 & 98.3 & 7.85 & 10.6 & 3.24 \\
\hline MONI & 13.48 & 93.6 & 0.81 & 96.5 & 5.92 & 11.2 & 0 \\
\hline
\end{tabular}

Valores porcentuales de los métodos de frecuencia de aparición (FA), abundancia específica de la presa en número (PN), numérico $(\mathrm{N})$, abundancia específica de la presa en peso $(\mathrm{PW})$, gravimétrico $(\mathrm{W})$ índice de importancia relativa específica de la presa (PSIRI) y nivel trófico de la presa (NT). Materia orgánica no identificada (MONI).

Percentages based on frequency of occurrence (FA), prey-specific number (PN), number (N), prey-specific weight (PW), weight (W), prey-specific index of relative importance (PSIRI) and trophic level of each prey category (NT). Unidentified organic matter (MONI).

C. vinctus $(\mathrm{n}=94)$, se registró un total de 19 recursos tróficos. De los cuales, se consideran los más importantes con base en el \% PSIRI a larvas mysis de Penaeoidea (32.67 \%) seguido por Euphausia sp. (27.3\%), otros Teleostei (11.23\%) y Anchoa ischana (9.36 \%) (Fig. 2).
Para el análisis estacional de los meses muestreados fueron divididos en dos temporadas del año (lluvias y secas). Para la temporada de lluvias se registraron 19 recursos tróficos en $C$. vinctus, siendo el principal las larvas megalopa de Portunoidae (25.6 \%), larvas 


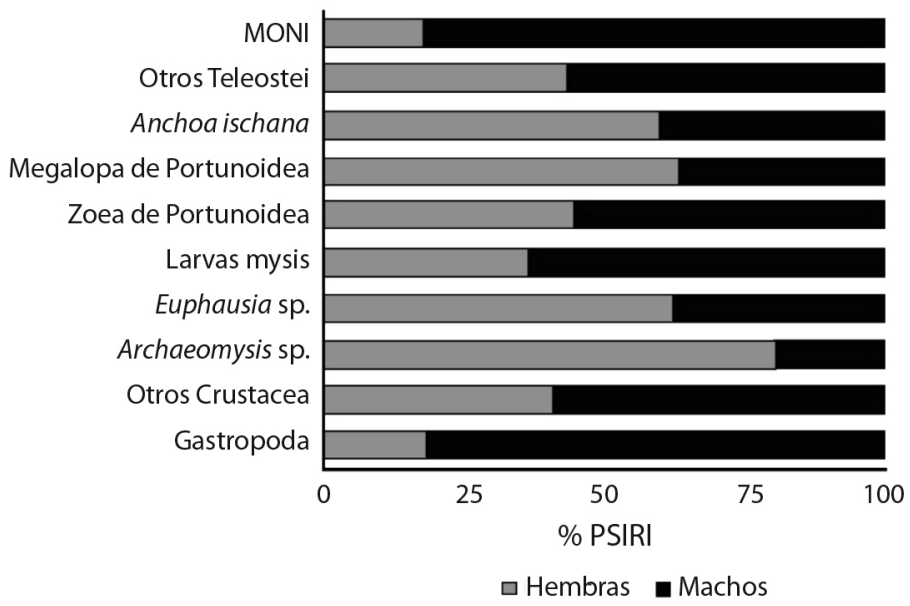

Fig. 1. Componentes de la dieta de C. vinctus de acuerdo al sexo (\% PSIRI).

Fig. 1. Components of the $C$. vinctus diet according to sex (\% PSIRI).

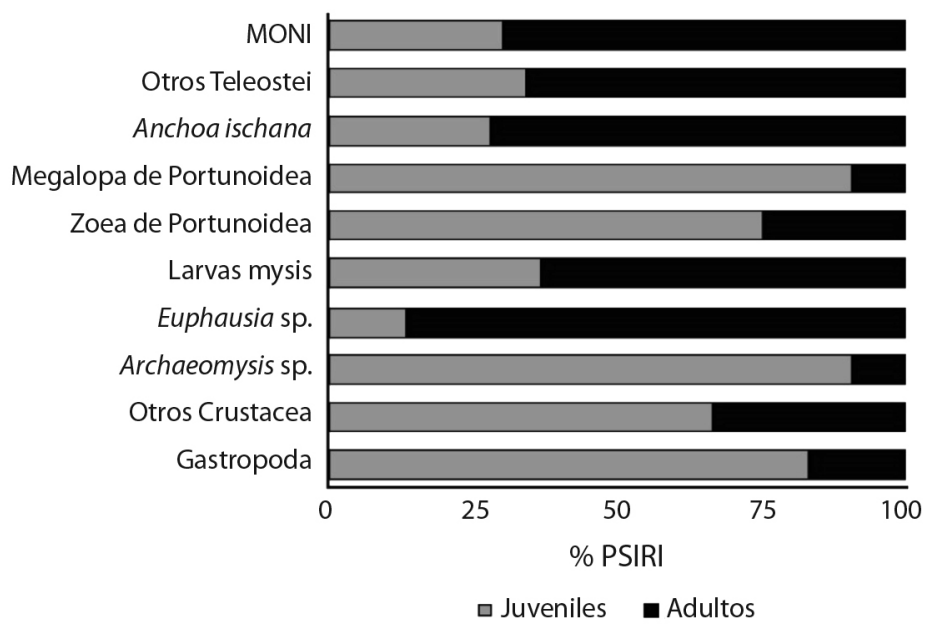

Fig. 2. Componentes de la dieta de $C$. vinctus de acuerdo a la madurez sexual (\% PSIRI).

Fig. 2. Components of the $C$. vinctus diet according to the sexual maturity (\% PSIRI).

mysis de Penaeoidea (25.02\%) Archaeomysis sp. (14.81\%) y otros Crustacea (13.53\%), conformando el $78.98 \%$ del índice de importancia relativa específica de la presa en un total de 143 estómagos llenos. Para la época de secas (precipitaciones menores a $15 \mathrm{~mm} / \mathrm{mes})$ los ítems más importantes fueron: Euphausia sp. (47.76 $\%)$, Anchoa ischana (32.83\%), MONI (6.34 $\%)$ y otros Teleostei $(5.67 \%)$, conformando el $92.61 \%$ del índice de importancia relativa específica de la presa en un total de 125 estómagos llenos (Fig. 3).
Al aplicar el índice de Levin se determinó que $C$. vinctus puede caracterizarse como especialista ya que el valor estimado de amplitud fue bajo $(\mathrm{Bi}=0.29)$ en el espectro trófico general. Por lo cual, se considera como un depredador, con marcadas preferencias hacia algunos ítems (megalopas de Portunoidae, Teleostei y Anchoa ischana). La estrategia especialista se mantuvo en los análisis realizados por categorías (sexo, madurez sexual y temporada) (Tabla 3). De acuerdo al índice de Morisita Horn, se presentó un traslape entre las dietas por sexo 


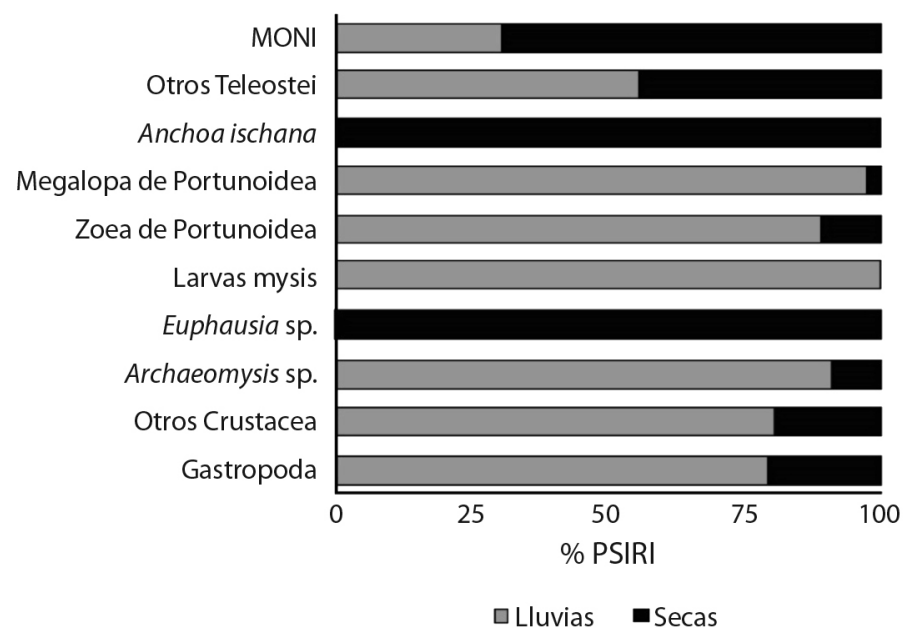

Fig. 3. Componentes de la dieta de $C$. vinctus de acuerdo a la época del año (\% PSIRI).

Fig. 3. Components of the $C$. vinctus diet according to the time of year (\% PSIRI).

TABLA 3

Amplitud de dieta de $C$. vinctus de acuerdo al índice de Levin. General, sexo, madurez sexual y época

TABLE 3

Diet breadth of $C$. vinctus according to the Levin index. General, sex, sexual maturity and time

\begin{tabular}{lc}
$\quad$ Categoría & Valor \\
General & 0.29 \\
Sexo & \\
$\quad$ Hembras & 0.32 \\
$\quad$ Machos & 0.34 \\
Madurez sexual & \\
$\quad$ Juveniles & 0.34 \\
$\quad$ Adultos & 0.21 \\
Época & \\
$\quad$ Lluvias & 0.28 \\
$\quad$ Secas & 0.12 \\
\hline
\end{tabular}

(hembras y machos $\mathrm{C} \lambda=0.84$ ), pero no entre madurez sexual (Juveniles y adultos $\mathrm{C} \lambda=0.56$ ) $\mathrm{y}$ temporadas (Lluvias y secas $\mathrm{C} \lambda=0.03$ ). La prueba ANOSIM demostró que en todas las combinaciones se registraron diferencias significativas $(\mathrm{P}<0.05)($ Tabla 4$)$.

De acuerdo con la ecuación de nivel trófico (NT) propuesta por Cortés (1999), se estableció que de manera general C. vinctus es una especie zooplantofaga e ictiófaga $(\mathrm{NT}=3.39)$, para secas $(\mathrm{NT}=3.15)$ y para lluvias $(\mathrm{NT}=$ 3.52). Lo que lo clasifica como un consumidor terciario. Este tipo de consumidores se caracterizan por consumir especies de segundo orden en la cadena alimenticia como son las anchoas, algunos crustáceos y zooplancton, es decir, de especies consumidoras de los grupos localizados en niveles inferiores de la cadena trófica.

TABLA 4

Estadístico R, valor de P y Similitud en la prueba ANOSIM entre sexos, madurez sexual y temporadas para Caranx vinctus en la Bahía de Acapulco, México

TABLE 4

R statistic, P-values and Similarity of ANOSIM test between sexes, sexual maturity, and seasons for Caranx vinctus from Acapulco Bay, Mexico

\begin{tabular}{lccc}
\multicolumn{1}{c}{ Grupo } & Estadístico global (R) & Nivel de significancia P & Similitud (\%) \\
Temporadas (lluvias y secas) & 0.810 & 0.001 & 49.5 \\
Madurez sexual (Juveniles y adultos) & 0.996 & 0.001 & 68.1 \\
Sexos (Hembras y machos) & 0.983 & 0.001 & 83.2 \\
\hline
\end{tabular}




\section{DISCUSIÓN}

Para C. vinctus, considerando el intervalo de tallas que componen las capturas (16 - 27.5 $\mathrm{cm}$ de LT), la pesquería artesanal de la bahía de Acapulco estaría compuesta principalmente de organismos inmaduros, ya que de acuerdo a Lucano-Ramírez, Rivera-Ríos, Ruiz-Ramírez, González-Sansón, y Pérez-Toledo (2016), la talla de primera madurez (L50) es de 26.04 $\mathrm{cm}$ para hembras y $23.77 \mathrm{~cm}$ para machos. De acuerdo al análisis de los 244 organismos a los cuales se les determino el sexo se estimó que su L50 es de $22 \mathrm{~cm}$ LT. Se considera que esta especie al estar en su aprovechamiento máximo presenta madurez temprana, e incluso se encontraron organismos de tallas de $17 \mathrm{~cm}$ de LT, con gónada desarrollada, esto posiblemente se debe a que los organismos capturados debido a la presión de pesca aceleran su crecimiento al disminuir la competencia intraespecífica por alimento y por tanto a menor talla alcanzan su madurez sexual (Longhurst \& Pauly, 1987).

El índice de importancia relativa (IIR) (Pinkas, Oliphant, \& Iverson, 1971) es uno de los índices más utilizados para determinar hábitos alimentarios de una especie, sin embargo en la actualidad se sabe que este índice conlleva desaciertos matemáticos y metodológicos, que pueden derivar en una interpretación errónea de los resultados. En atención a dicha problemática Brown et al. (2012), propusieron el uso del índice de importancia relativa específico de las presas (\% PSIRI), mismo que pondera la importancia de cada artículo alimentario únicamente entre los organismos en que se presentó, de esta forma se obtiene mayor precisión de la dieta y se evitan sesgos de sobre o subestimación de presas, contrario a lo que ocurre cuando se opta por el \% IIR, ya que pondera la importancia de cada presa con respecto a los estómagos que presentaron contenido (Moreno-Sánchez, et al., 2019).

Los estómagos analizados permitieron identificar 24 ítems alimentarios. Los resultados de este trabajo indican que, en la bahía de Acapulco, Guerrero C. vinctus se alimenta de una gran variedad de presas zooplanctónicas, representadas por crustáceos y huevos y larvas de peces, así como pequeños peces de la familia Engraulidae. Otras especies de la familia Carangidae que también basan su alimentación en crustáceos y peces se han considerado como depredadores especialistas (Roux \& Conand, 2000; Sley, Jarboui, Ghorbel, \& Bouain, 2008; Flores-Ortega et al., 2010; Sánchez-García et al. 2017).

Los datos de este trabajo coinciden con lo reportado por De la Cruz-Agüero et al. (1997). Según estos autores para Baja California Sur, México, $C$. vinctus se alimenta principalmente de pequeños peces, crustáceos e invertebrados bentónicos. En la Bahía de Acapulco los peces pequeños estuvieron representados por Anchoa ischana $(\%$ PSIRI $=10.6 \%)$, los crustáceos planctónicos principalmente por larvas zoea de Porcellanidae, megalopas de Portunoidae, larvas mysis de Penaeoidea y Euphausia sp. que conformaron el $39.41 \%$ del \% PSIRI. Un crustáceo bentónico intersticial fue Archaeomysis sp. que represento el $5.5 \%$ del \% PSIRI. Otros invertebrados bentónicos presentes en la dieta de $C$. vinctus fueron: Turritellidae, Littorinidae, Cirripedia, Squilla sp. y Axiidae sp. que en conjunto conformaron el $2.19 \%$ del $\%$ PSIRI. En general para estudios tróficos se debe tomar en cuenta que el grado de diversidad de hábitos alimentarios en peces, deriva de una serie de procesos coevolutivos entre estos y sus presas en respuesta a diferentes condiciones ambientales. Dichos procesos implican amplia gama de adaptaciones a nivel etológico, metabólico y anatómico en orden de un mejor aprovechamiento de los recursos tróficos (Granado-Lorencio, 2002), ya que generalmente seleccionan presas que maximizan la cantidad de energía ganada, en relación al coste energético por captura (Sánchez-Hernández, Servia, Vieira-Lanero, \& Cobo, 2013) y siempre tratando de reducir en lo posible la competencia con otras especies por el alimento (GranadoLorencio, 2002).

$\mathrm{Al}$ parecer $C$. vinctus, forma cardúmenes mixtos, por lo que no hay una segregación por sexos en la alimentación $(\mathrm{C} \lambda=0.84)$. Por otro lado, de acuerdo al índice de Morisita Horn hay 
poco traslape entre la dieta de juveniles y adultos $(C \lambda=0.56)$, este resultado sugiere que en $C$. vinctus existe un patrón en la utilización de los recursos alimentarios de acuerdo a las distintas clases de talla, como una posible estrategia ecológica para la optimización en el uso de las presas para minimizar la competencia intraespecífica (Schoener, 1971; Cortés \& Gruber, 1990; Ruiz-Campos \& Cota-Serrano, 1992; Vögler, Milessi, \& Quiñones, 2003).

En este análisis el espectro alimentario de $C$. vinctus exhibió una variación estacional cualitativa y cuantitativa (Lluvias y secas $\mathrm{C} \lambda$ $=0.03$ ). A lo largo del período de estudio se encontraron diferentes grupos de organismos en el contenido estomacal. Para la temporada de lluvias, $C$. vinctus se alimentó principalmente de larvas megalopa de Portunoidae (25.6\%) y no apareció en la dieta Euphausia sp., para la época de secas el ítem más importante fue Euphausia sp. (47.76 \%), en este periodo estuvieron ausentes larvas mysis de Penaeoidea. La diferencia en la alimentación entre las temporadas de lluvias y secas, está relacionada con variaciones en la disponibilidad del alimento, debido a cambios en la temperatura, nutrientes, movimientos de surgencias locales e interacciones oceánicas dentro de la Bahía de Acapulco (Meave-del Castillo, Zamudio-Resendiz, \& Castillo Rivera, 2012; Rojas-Herrera et al., 2012, Fernández-Álamo, Gálvez-Zeferino, Roa-Venicio, \& Rojas-Herrera, 2019). En el Mediterráneo, C. rhonchus Geoffroy SaintHilaire, 1817 y C. crysos (Mitchill, 1815) presenta diferencias estacionales en su dieta, presentando una mayor incidencia de crustáceos durante otoño-invierno en su dieta, y en verano presenta un aumento en el consumo de peces (Sley et al. 2008, 2009), lo que coincide con este estudio, donde el consumo de crustáceos disminuye en época de secas y aumenta en lluvias. También se observó una mayor incidencia de Anchoa ischana en época de secas, lo cual coincide con lo observado en el Golfo de California (Sánchez-García et al. 2017), en donde Anchoa spp. fue más abundante en la dieta del jurel (C. caninus Günther, 1867) durante la época fría. Además, similarmente a lo reportado por Sánchez-García et al. (2017) en C. vinctus aumenta la diversidad de sus presas en época de secas, principalmente crustáceos.

Para una comparación estadística más rigurosa de la alimentación se empleó en este trabajo el análisis multivariado de similitud (ANOSIM), el cual mostró que los factores (sexo, madurez sexual y temporada) influyeron en el tipo de alimentación de $C$. vinctus.

El resultado obtenido con el índice de Levin indica que $C$. vinctus se alimenta de un reducido número de organismos presas, mostrando una amplitud de dieta baja $(\mathrm{Bi}=0.29)$ por lo que se le clasificó como depredador especialista oportunista, pues cambia su dieta de acuerdo a las presas más abundantes en un tiempo y espacio específico. La doradita puede alimentarse de un grupo limitado de organismos aprovechando las presas que están disponibles en el área. Un comportamiento parecido en la dieta especialista se ha observado en otros carangidos como Selar crumenophthalmus (Bloch, 1793), Selene peruviana (Guichenot, 1866) y Caranx caninus (Roux \& Conand, 2000; Tripp-Valdez \& Arreguín-Sánchez, 2012; Sánchez-García et al., 2017). Cuando existe una abundancia del recurso los depredadores tienen una selección por ciertas presas, es decir, les permite elegir a sus presas, en caso contrario, cuando el recurso es escaso, los depredadores consumen las presas sin ninguna selección (Escobar-Sánchez, Abitia-Cárdenas, \& Galván-Magaña, 2006).

Palacios-Salgado (2011) para comunidades de peces de fondos blandos en el Pacifico Oriental Tropical de México en cinco zonas típicas de pesca de camarón, encontró 44 grupos funcionales (incluyendo gremios tróficos y gremios reproductivos) aplicando descriptores estructurales como la composición, la diversidad taxonómica y la afinidad biogeográfica; así como datos asociados a hábitat, como son uso de sistemas estuarinos, posición en la columna de agua y distribución vertical, y descriptores morfológicos como son la forma del pez y de la aleta caudal, entre otros. La doradita $C$. vinctus fue agrupada con Caranx caballus Günther, 1868, C. caninus, C. otrynter Jordan \& Gilbert, 
1883 y Scomberomorus sierra Jordan \& Starks, 1895. Los atributos que definen a este grupo son, ictiobentívoros, nivel trófico 3.5 - 3.99, especies pelágico neríticas, cuerpo fusiforme, ovíparos con huevos pelágicos, aleta caudal furcada y teleósteos grandes. Estas cinco especies han sido reportadas en Acapulco, Guerrero, México (Palacios-Salgado, RamírezValdez, Rojas-Herrera, Granados-Amores, \& Melo-García, 2014) por lo que este grupo funcional está completo en el área de estudio.

Declaración de ética: los autores declaran que todos están de acuerdo con esta publicación y que han hecho aportes que justifican su autoría; que no hay conflicto de interés de ningún tipo; y que han cumplido con todos los requisitos y procedimientos éticos y legales pertinentes. Todas las fuentes de financiamiento se detallan plena y claramente en la sección de agradecimientos. El respectivo documento legal firmado se encuentra en los archivos de la revista.

\section{AGRADECIMIENTOS}

Al Consejo Nacional de Ciencia y Tecnología (CONACYT) por la beca parcial otorgada a María Isabel Vázquez-Ozuna para estudios de posgrado y a los evaluadores anónimos por las valiosas observaciones realizadas para la mejora del presente trabajo.

\section{RESUMEN}

Introducción: Caranx vinctus es una especie importante en las pesquerías locales, utilizada como fuente de proteína para el consumo humano, sin embargo, su ecología trófica es poco conocida en las costas de Pacífico mexicano. Objetivo: Conocer los hábitos alimenticios de C. vinctus. Metodología: Se analizaron los contenidos estomacales de 268 ejemplares, capturados mediante el arte de pesca, chinchorro playero, en la sociedad cooperativa Playa las Hamacas, en la Bahía de Acapulco, Guerrero, México. Se aplicó el índice de importancia relativa específica de la presa (\% PSIRI) para determinar las principales categorías alimenticias (ítems). La amplitud de la dieta se calculó utilizando el índice de Levin. Las posibles diferencias en la dieta por sexo, madurez sexual o temporada se identificaron a través del índice Morisita Horn y un análisis multivariado ANOSIM. Resultados: Los principales ítems fueron crustáceos representados principalmente por larvas megalopa de Portunoidae (16.51\%), Teleostei (12.7\%), materia orgánica no identificada (11.2\%), y Anchoa ischa$n a(10.6 \%)$ El índice de Levin indica baja amplitud del nicho trófico $(\mathrm{Bi}=0.29)$ por lo que se le puede considerar como un depredador especialista con marcadas preferencias hacia algunos tipos alimentarios. El índice de Morisita Horn indicó que, se presentó un traslape entre las dietas por sexo (hembras y machos $\mathrm{C} \lambda=0.84$ ), pero no entre madurez sexual (Juveniles y adultos $\mathrm{C} \lambda=0.56$ ) y temporadas (Lluvias y secas $C \lambda=0.03)$. Un análisis ANOSIM indicó que había diferencias significativas en la dieta de $C$. vinctus por sexo, madurez sexual y temporada. Conclusión: Se estableció que de manera general $C$. vinctus es una especie zooplantófaga e ictiófaga (Nivel Trófico $=3.39$ ).

Palabras clave: jureles; Carangidae; dieta; nivel trófico; Portunoidae; Anchoa ischana; Pacífico Oriental Tropical.

\section{REFERENCIAS}

Allen, G.R., \& Robertson, D.R. (1998). Peces del Pacifico oriental tropical. México D. F.: CONABIO, Agrupación Sierra Madre y CEMEX.

Brown, S.C, Bizzarro, J.J., Cailliet, G.M., \& Ebert, D.A. (2012). Breaking with tradition: redefining measures for diet description with a case study of the Aleutian skate Bathyraja aleutica (Gilbert 1896). Environmental Biology of Fishes, 95(1), 3-20. DOI: 10.1007/ s10641-011-9959-z

Brusca, R.C. (1980). Common intertidal invertebrates of the Gulf of California. Tucson, Arizona: University of Arizona Press.

Clarke, K.R., \& Warwick, R.M. (2001). Change in marine communities: an approach to statistical analysis and interpretation. Plymouth: Natural Environment Research Council, Plymouth Marine Laboratory.

Colwell, R. (2016). Estimates: Statistical estimation of species richness and shared species from samples (Version 9.1.0). Recuperado de http://viceroy.eeb. uconn.edu/Estimates

Correa, C.E., Albrecht, M.P., \& Hahn, N.S. (2011). Patterns of niche breadth and feeding overlap of the fish fauna in the seasonal Brazilian Pantanal, Cuiabá River basin. Neotropical Ichthyology, 9(3), 637-646.

Cortés, E. (1999). Standardized diet compositions and trophic levels of sharks. ICES. Journal of Marine Science, 56, 707-717.

Cortés, E., \& Gruber, S.H. (1990). Diet, feeding habits and estimates of daily ration of Young lemon sharks, Negaprion brevirotris (Poey). Copeia, 1, 204-218. 
Cury, P., Bakun, A., Crawford, R.J.M., Jarre-Teichmann, A., Quinones, R., Shannon, L.J., \& Verheye, H.M. (2000). Small pelagics in upwelling systems: patterns of interaction and structural changes in "waspwaist" ecosystems. ICES Journal of Marine Science, 57, 603-618.

Dantas, D.V., Barletta, M., Ramos, J. de A.A., Lima, A.R.A., \& da Costa, M.F. (2013). Seasonal diet shifts and overlap between two sympatric catfishes in an estuarine nursery. Estuaries and Coasts, 36, 237-256.

De la Cruz-Agüero, J., Arellano-Martínez, M., CotaGómez, V.M., \& de la Cruz-Agüero, G. (1997). Catálogo de los peces marinos de Baja California Sur. La Paz, Baja California Sur, México: Instituto Politécnico Nacional-Centro Interdisciplinario de Ciencias Marinas.

Escobar-Sánchez, O., Abitia-Cárdenas, L.A., \& GalvánMagaña, F. (2006). Food habits of the Pacific angel shark Squatina californica in the southern Gulf of California. Cybium, 30(4), 91-97.

Espino-Barr, E., Cruz, M., \& García, A. (2003). Peces marinos con valor comercial de la costa de Colima, México. México: CONABIO, Instituto Nacional de Pesca.

Estupiñán-Montaño, C., Cedeño-Figueroa, L.G., \& Galván-Magaña, F. (2009). Hábitos alimentarios del tiburón martillo Sphyrna lewini (Griffith \& Smith, 1834) (Chondrichthyes) en el Pacífico ecuatoriano. Revista de Biología Marina y Oceanografía, 44(2), 379-386. DOI: 10.4067/S0718-19572009000200011

Fernández-Álamo, M.A., Gálvez-Zeferino, N., RoaVenicio, M., \& Rojas-Herrera, A. (2019). Variación espacio-temporal de diversidad y densidad de los poliquetos pelágicos en la Bahía de Acapulco, Guerrero, México. Revista de Biología Tropical, 67(5), 92-100.

Fischer, W., Krupp, F., Schneider, W., Sommer, C., Carpenter, K.E., \& Niem, V.H. (Eds.) (1995). Pacífico Centro-Oriental; Guía FAO para la identificación de especies para los fines de pesca. Roma, Italia: Food and Agriculture Organization of the United Nations (FAO).

Flores Ortega, J.R., Godínez Domínguez, E., Rojo Vázquez, J.A., Corgos, A., Galván Piña, V.H., \& González Sansón, G. (2010). Interacciones tróficas de las seis especies de peces más abundantes en la pesquería artesanal en dos bahías del Pacífico Central Mexicano. Revista de Biología Tropical, 58(1), 383-397.

García-Madrigal, M.D.S. (2007). Annotated check list of the amphipods (Peracarida: Amphipoda) from the tropical eastern Pacific. Contributions to the study of East Pacific Crustaceans, 4(2), 63-195.

Granado-Lorencio, C. (2002). Ecología de peces. Sevilla, España: Universidad de Sevilla.
Guedes, A.P.P., Araujo, F.G., Pessanha, A.L.M., \& Milagre, R.R. (2015). Partitioning of the feeding niche along spatial, seasonal, and size dimensions by the fish community in a tropical bay in southeastern Brazil. Marine Ecology, 36, 38-56.

Gutiérrez-Zavala, R.M., \& Cabrera-Mancilla, E. (2019). Especies marinas de valor comercial en el estado de Guerrero. México: Instituto Nacional de Pesca y Acuacultura.

Hajisamaea, S., Choua, L.M., \& Ibrahim, S. (2003). Feeding habits and trophic organization of the fish community in shallow waters of an impacted tropical habitat. Estuarine, Coastal and Shelf Science, 58, $89-98$

Hendrickx, M.E., \& Salgado-Barragán, J. (1991). Los estomatópodos (Crustacea: Hoplocarida) del Pacífico Mexicano. México: Universidad Nacional Autónoma de México, Instituto de Ciencias del Mar y Limnología.

Hoffmayer, E.R., \& Parsons, G.R. (2003). Food habits of three shark species from the Mississippi sound in the northern Gulf of Mexico. Southeastern Naturalist 2(2), 271-280.

Horn, H.S. (1966). Measurement of overlap in comparative ecological studies. American Naturalist, 100, 419-424.

Hynes, H.B.N. (1950). The food of freshwater sticklebacks (Gasterosteus aculeatus and Pygosteus pungitius), with a review of methods used in studies of the food of fishes. Journal of Animal Ecology, 19, 35-38.

Hyslop, E.J. (1980). Stomach contents analysis. A review of methods and their application. Journal of Fish Biology, 17, 411-429.

Jiménez-Valverde, A., \& Hortal, J. (2003). Las curvas de acumulación de especies y la necesidad de evaluar la calidad de los inventarios biológicos. Revista Ibérica de Aracnología, 8, 151-161.

Júnior, T.V., Vooren, C.M., \& Lessa, R.P. (2004). Feeding habits of four species of Istiophoridae (Pisces: Perciformes) from northeastern Brazil. Environmental Biology of Fishes, 70, 293-304.

Keen, A.M. (1958). Sea shells of tropical West America. Stanford: Stanford University.

Krebs, C.J. (1999). Ecological methodology. New York USA: Harper \& Row.

Langton, R.W. (1982). Diet overlap between the Atlantic cod Gadus morhua, silver hake Merluccius bilinearis and fifteen other northwest Atlantic finfish. U.S. National Marine Fisheries Service. Fishery Bulletin, 80, 745-759. 
Longhurst, A., \& Pauly, D. (1987). Ecology of Tropical Oceans. San Diego, USA: Academic Press.

Lucano-Ramírez, G., Rivera-Ríos, E.G., Ruiz-Ramírez, S., González-Sansón, G., \& Pérez-Toledo, A. (2016). Reproducción de Carangoides vinctus (Perciformes: Carangidae) en el Pacífico central mexicano. Latin American Journal of Aquatic Research, 44(3), 610622. DOI: $10.3856 /$ vol44-issue3-fulltext-20

Mauchline, J. (1971). Euphausiacea larvae. Copenhagen: Conseil International pour l'Exploration de la Mer ICES. DOI: https://doi.org/10.17895/ices.pub.5110

Meave-del Castillo, M.E., Zamudio-Resendiz, M.E., \& Castillo Rivera, M.A. (2012). Riqueza fitoplanctónica de la Bahía de Acapulco y zona costera aledaña, Guerrero, México. Acta Botánica Mexicana, 100, 405-487. DOI: doi. org/10.21829/abm100.2012.41

Modlin, R.F. (1984). Mysidacea from the Florida Middle Ground, northeast Gulf of Mexico, with descriptions of three new species of Heteromysis and a key to the Heteromysini of the western Atlantic. Journal of Crustacean Biology, 4(2), 278-29.

Moreno-Sánchez, X.G., Pérez-Rojo, P., Irigoyen-Arredondo, M.S., Marín-Enríquez, E., Abitia-Cárdenas, L.A., \& Escobar-Sánchez, O. (2019). Feeding habits of the leopard grouper, Mycteroperca rosacea (Actinopterygii: Perciformes: Epinephelidae), in the central Gulf of California, BCS, México. Acta Ichthyologica et Piscatoria, 49(1), 9-22. DOI: 10.3750/AIEP/02321

Oxenford, H.A., \& Hunte, W. (1999). Feeding habits of the solphinfish (Coryphaena hippurus) in the Eastern Caribbean. Scientia Marina, 63(3-4), 303-315.

Palacios-Salgado, D.S. (2011). Patrones latitudinales de composición y diversidad funcional de peces asociados a la pesca de camarón del Pacífico mexicano (Tesis Doctoral). Instituto Politécnico Nacional, La Paz, Baja California Sur, México.

Palacios-Salgado, D.S., Ramírez-Valdez, A., Rojas-Herrera, A.A., Granados-Amores, J., \& Melo-García, M.A (2014). Marine fishes of Acapulco, Mexico (Eastern Pacific Ocean). Marine Biodiversity, 44(4), 471-490.

Pauly, D., Zeller, D., \& Palomares, M.L.D. (Eds.) (2020) Sea around Us Concepts, Design and Data. Recuperado de http://www.seaaroundus.org/data/\#/ marine-trophic-index

Pinkas, L., Oliphant, M.S., \& Iverson, L.K. (1971). Foods habits of albacore, bluefish tuna, and bonito in California Waters. California, USA: California Department of Fish and Game Fishery Bulletin, 152. Recuperado de http://escholarship.org/uc/ item $/ 7 \mathrm{t} 5868 \mathrm{rd}$

Ramos, D.M.A., Ferreira, A., dos Santos, N., Cunha, R.E.M.C., \& Machado, A. L. (2015). Trophic relationships among fish assemblages in a mudflat within
Brazilian marine protected area. Brazilian Journal of Oceanography, 63(2), 135-146.

Robertson, D.R., \& Allen, G.R. (2015). Peces Costeros del Pacifico Oriental Tropical: Sistema de Información en línea (Versión 2.0). Balboa, República de Panamá: Instituto Smithsonian de Investigaciones Tropicales. Recuperado de www.neotropicalfishes.org/sftep

Rojas-Herrera, A.A., Violante-González, J., García-Ibáñez, S., Sevilla-Torres, V.M.G., Gil-Guerrero, J.S., \& Flores-Rodríguez, P. (2012). Temporal variation in the phytoplankton community of Acapulco Bay, Mexico. Microbiology Research, 3(1), 13-19. DOI: doi. org/10.4081/mr.2012.e4

Roux, O., \& Conand, F. (2000). Feeding habits of the big eyes cad, Selar crumenophthalmus (Carangidae) in La Reunion Island waters (South-Western Indian Ocean). Cybium, 24(2), 173-179.

Ruiz-Campos, G., \& Cota-Serrano, P. (1992). Ecología alimenticia de la trucha arcoiris (Oncorhynchus mykiss nelsoni) del arroyo San Rafael, sierra San Pedro Mártir, Baja California, México. The Southwestern Naturalist, 37(2), 166-177.

Sánchez-García, C., Escobar-Sánchez, O., Valdez-Pineda, M.C., Ramírez-Pérez, J.S., Morán-Angulo, R.E., \& Moreno-Sánchez, X.G. (2017). Selective predation by crevalle jack Caranx caninus on engraulid fishes in the SE Gulf of California, Mexico. Environmental Biology of Fishes, 899-912. DOI: https://doi. org/10.1007/s10641-017-0615-0

Sánchez-Hernández, J., Servia, M.J., Vieira-Lanero, R., \& Cobo, F. (2013). Ontogenetic dietary shifts in a predatory freshwater fish species: the brown trout as an example of a dynamic fish species. En $\mathrm{H}$. Türker (Ed.), New advances and contributions to fish biology (pp. 271-298). Croatia: TechOpen. Recuperado de https://www.intechopen.com/books/ new-advances-and-contributions-to-fish-biology

Saucedo-Lozano, M., Bernal-Ornelas, I.H., Espino-Barr, E., García-Boa, A., Cabral-Solís, E.G., \& PuenteGómez, M. (2012). Feeding habits of the green jack (Caranx caballus Günther, 1868) on the coast of Manzanillo, Colima, México. The Open Marine Biology, 6, 28-37.

Schoener, T.W. (1971). Theory of feeding strategies. Annual Review of Ecology, Evolution, and Systematics, 2, 369-404.

Silvano R.A.M. (2001). Feeding habits and interspecific feeding associations of Caranx latus (Carangidae) in a subtropical reef. Environmental Biology of Fishes, $60,465-470$.

Sley, A., Jarboui, O., Ghorbel, M., \& Bouain, A. (2009). Food and feeding habits of Caranx crysos from the Gulf of Gabès (Tunisia). Journal of the Marine 
Biological Association of the United Kingdom, 89(7), 1375-1380.

Sley, A., Jarboui, O., Ghorbel, M., \& Bouain, A. (2008) Diet composition and food habits of Caranx rhonchus (Carangidae) from the Gulf of Gabes (central Mediterranean). Journal of the Marine Biological Association of the United Kingdom, 88(4), 831-836.

Smith-Vaniz, W.F. (1995). Carangidae. Jureles, pámpanos, cojinúas, zapateros, cocineros, casabes, macarelas, chicharros, jorobados, medregales, pez piloto. En W. Fischer, F. Krupp, W. Schneider, C. Sommer, K.E. Carpenter, \& V. Niem (Eds.). Guia FAO para la Identificación de Especies para los Fines de la Pesca. Pacifico Centro-Oriental (pp. 940-986). Roma: FAO.

Smith, P.E., \& Zaret, M.T. (1982). Bias in estimating niche overlap. Ecology, 12, 1248-1253.
Tripp-Valdez, A., \& Arreguín-Sanchez, F. (2012). The food of Selene peruviana (Actinopterygii: Perciformes: Carangidae) in the southern Gulf of California. Acta Ichthyologica et Piscatoria, 42(1), 1-7.

tutiempo.net (2019) Datos reportados por la estación meteorológica: 768050. Latitud: 16.83 | Longitud: -99.93 | Altitud: 28. Recuperado de https://www. tutiempo.net/clima/12-2019/ws-768050.html

Vögler, R., Milessi, A.C., \& Quiñones, R.A. (2003). Trophic ecology of Squatina guggenheim on the continental shelf off Uruguay and northern Argentina. Journal of Fish Biology, 62, 1254-1267.

Yáñez-Arancibia, A., \& Nugent, R. (1977). El papel ecológico de los peces en estuarios y lagunas costeras. Anales del Instituto de Ciencias del Mar y Limnología, 4, 107-117. 